\title{
CIUDAD SUSTENTABLE Y ACCESO AL TRANSPORTE PÚBLICO El caso de una ciudad intermedia argentina
}

\author{
Ana Laura Castañeda Nordmann \\ Universidad o Institución a la que pertenece: Centro de Estudios del Territorio y el Hábitat Popular (CETyHaP), \\ Facultad de Arquitectura y Urbanismo (FAU), Universidad Nacional de Tucumán (UNT) \\ Director de la investigación: Claudia Fernanda Gómez López \\ anuchicn@hotmail.com
}

\section{RESUMEN}

Los patrones latinoamericanos de movilidad no han traspasado los modelos instaurados en la modernidad, ya que si bien el estudio sobre ésta temática cada vez es mayor, las políticas públicas actuales no logran un cambio de matriz energética, lo que conlleva a un costo ambiental muy alto e insustentabilidad urbana. Asimismo la dotación de áreas verdes públicas no se relaciona a los mayores requerimientos de disminución de $\mathrm{CO}_{2}$, es por ello que el trabajo tiene como finalidad identificar en el Área Metropolitana de Tucumán la emisión de $\mathrm{CO}_{2}$ del transporte público en relación a la presencia de actividad fotosintética que permita contrarrestar niveles altos de $\mathrm{CO}_{2}$ emitidos por el uso diario del transporte. Se utiliza una metodología en SIG para la obtención de áreas críticas que permitan generar propuestas de intervención. Los resultados alcanzados evidencian la importancia de incorporar medidas desde las políticas públicas para aumentar las condiciones de habitabilidad.

Palabras clave: Posmodernidad, sustentabilidad, movilidad pública urbana

\begin{abstract}
The Latin American patterns of mobility have not transcended the models established in modernity, because although the study on this topic is growing, current public policies do not achieve a change in the energy matrix, which leads to a very high environmental cost and urban unsustainability. Likewise, the endowment of public green areas is not related to the greater requirements of $\mathrm{CO}_{2}$ reduction, that is why the work has the purpose of identifying in the Metropolitan Area of Tucumán the emission of $\mathrm{CO}_{2}$ from public transport in relation to the presence of photosynthetic activity that allows to counteract high levels of $\mathrm{CO}_{2}$ emitted by the daily use of transport. A GIS methodology is used to obtain critical areas that allow generating intervention proposals. The results achieved demonstrate the importance of incorporating measures from public policies to increase living conditions.
\end{abstract}

Key words: Postmodernity, sustainability, urban public mobility 


\section{INTRODUCCIÓN}

Comprender la realidad urbana desde la existencia de paradigmas epocales ${ }^{1}$ significa decodificarla a partir de fundamentos o modelos que sirven para identificar problemas y métodos, y para llevar a cabo determinadas prácticas que se trasfieren a sucesivas generaciones (Kuhn, 1971). Dichas leyes o teorías sirven para describir y explicar la realidad; como así también, para efectuar y predecir relaciones constantes entre los diferentes fenómenos (Pardo, 2000).

Se entiende que la estructura de pensamiento de la posmodernidad ha incentivado la crítica y reconceptualización de aspectos claves como el de habitabilidad urbana. En ésta, se han incorporado cuestiones como el sentido de pertenencia (sentirse parte), apropiación (hacerlo propio), diversidad (encontrar las ventajas de la diferencia), riesgo (prevenir), incertidumbre (comprender que las externalidades son una parte importante de la realidad); y lo que implica el habitar un lugar (material y simbólicamente), desde el análisis de las necesidades reales de la población y el costo energético que implica un confort urbano (Castañeda, 2017).

La política pública sin embargo, no ha podido traspasar los modelos instaurados en la modernidad ${ }^{2}$. En este modelo de pensamiento, la vida se traduce a una idea antropocentrista, con una concepción del espacio infinita e ilimitada. Se atribuye al conocimiento científico el carácter de certero e incuestionable, con una transmisión de la verdad mediante afirmaciones fuertes y universales. El saber se conforma por un nuevo sistema de creencias, basado en un orden racional y en la comprensión de un orden natural, a partir de caracteres o principios lógicos matemáticos. Se formula de esta forma, un conocimiento de validez universal, que vincula el progreso social al desarrollo de la ciencia y en el que conceptos como ética y moral son válidos para todos y se fundan en la pura razón o verdad (Pardo, 2000).

En este sentido, el acceso $^{3}$ al transporte público constituye un indicador -inherente al estado desde la representación como mandato- de medición de calidad de vida de la población al otorgar bienestar emocional y material; relaciones armónicas familiares, sociales y con el ambiente; actividad productiva; seguridad; autoestima social e integración con la comunidad (Velázquez, 2001; Ardila, 2003; Satriano, 2006; Hernández Aja, 2009; Tonón y Castro Solano, 2012). De manera que entender a los equipamientos que persiguen el bienestar social como indicadores de acceso urbano, implica asociarlos a las condiciones y niveles de satisfacción (calidad de vida) o carencia (pobreza) relacionados al bienestar físico, psicológico, social, material y ambiental (Velázquez, 2001; Longhi et al., 2015).

El acceso al transporte público, entendido como elemento de servicio funcional urbano cotidiano -colectivo y masivo- de desenvolvimiento y aceleración de la vida social, otorga satisfacción en tanto posibilita el traslado simultáneo de grandes volúmenes de carga de personas por corredores de gran densidad de demanda desde un aumento del tiempo invertido- al trabajo, centros de abastecimiento y consumo, equipamientos (educativos, sanitarios, recreativos, entre otros); y la realización de diversas actividades (Figueroa, 2005; Rodríguez Vignoli, 2008; Navarrete Rodríguez y Andrade Vallejo, 2010; Lange Valdes, 2011; Dangond Gibsone et al., 2011). A su vez, aporta como herramienta de control y organización del espacio a los procesos de transformación, reestructuración y expansión urbana y desde la relación con el entorno por el que circula a la conectividad de las diferentes áreas y producción del espacio urbano, a través de estimular la concentración de actividades e intervenir en la valorización diferencial del suelo (Gutiérrez, 2000; Figueroa, 2005; Lange Valdes, 2011; Blanco et al., 2014).

Por otro lado, la dotación de espacios verdes públicos también constituye un aspecto clave a tener en cuenta, desde las políticas públicas, para aumentar las condiciones de habitabilidad urbana de la población. Estos son definidos como lugares abiertos de uso libre, pertenencia social y dominio estatal; que constituyen -como componentes básicos de la matriz urbana- un recurso estratégico para el desarrollo de la ciudad en términos de beneficios ambientales y fomento del ocio, estancia, esparcimiento y salubridad social (Hernández Aja y Leiva Rodríguez, 2006; Di Lullo, 2009). Constituyen, a la vez, espacios que generan centralidad de servicios y calidad de vida urbana, y requieren de una determinada accesibilidad para sostenerse (Castañeda, inédito). En este contexto, un indicador utilizado actualmente para identificar niveles de calidad de vida de la población es la presencia de parques y espacios verdes, entendidos como recursos recreativos. Dicho indicador es comprendido -desde la perspectiva de oportunidad- en el de Calidad de Vida (ICV) elaborado por Velázquez y Celemín (2013) para el año 2010 y -desde la evaluación de niveles de satisfacción- en el Índice Sintético

\footnotetext{
${ }^{1}$ Pardo (2000) hace referencia a tres tipos de paradigmas epocales. El primero, denominado Premoderno, el cual abarca desde el siglo VI a.C. hasta el siglo XV d.C. y se constituye por la Antigüedad clásica y la Edad Media. El segundo, denominado Moderno, que abarca desde el siglo XVI hasta mediados del siglo XX. Por último, el Posmoderno, que abarca desde mediados del siglo XX hasta la actualidad. ${ }^{2}$ Se caracteriza al paradigma moderno, como un período de grandes transformaciones sociales y técnicas, que derivan en sucesivas crisis sociales y científicas (De Sousa Santos, 2009).

${ }^{3}$ El acceso es comprendido desde la disponibilidad de la población de materializar sus necesidades (Pardo, 2005).
} 
de Condiciones de Vida Urbano (ISCVU) elaborado por Boldrini et al. (2014); sin embargo no es tenido en cuenta -desde la evaluación de niveles de carencia- en el de Vulnerabilidad Social (IVS) elaborado por Paolasso et al. (en prensa). La importancia de estos dos últimos radica en que constituyen Índices de aplicación en el Área Metropolitana de Tucumán y, en este sentido, si se analiza dicha cobertura de espacios verdes la población en condiciones de vulnerabilidad evidencia mayor dificultad de acceso.

En relación a esto, la calidad de vida considera las experiencias subjetivas de los individuos que la integran y que tienen de su existencia en la sociedad; y exige, en consecuencia, conocer cómo viven los sujetos (sus condiciones objetivas de existencia y expectativas de transformación que desean) y evaluar el grado de satisfacción que se consigue (Celemín, 2007). Así pues, su conceptualización se concibe como una construcción imprecisa (Gómez López, 1994), compleja y multifactorial (Velázquez, 2001; Celemín, 2007) sobre la que pueden desarrollarse algunas formas de medida objetivas a través de una serie de indicadores, pero donde adquiere un peso importante la vivencia que el sujeto pueda tener de sí mismo y de la realidad (Levi y Anderson, 1980; Celemín, 2007).

En la valoración del componente subjetivo entran en juego una serie de elementos, en un principio relacionados con las necesidades del individuo y posteriormente a las necesidades sociales y comunitarias (Levi y Anderson, 1980). Estos autores consideran imprescindible el establecimiento de un estándar colectivo, aunque aclaran que únicamente es válido para el momento y contexto específico de su establecimiento. Todo esto conlleva a poder conceptualizar la calidad de vida como una adaptación entre las características de la situación de la realidad y las expectativas, capacidades y necesidades del individuo, tal como las percibe él mismo y su grupo social.

Analizar la calidad de vida en la ciudad requiere, según Chombart de Lauwe (1976), de una postura ideológica de partida, la cual conlleva a una valoración del contexto de la salud (en su aspecto comunitario, médico, asistencial y cualitativo) y de la interacción social (en el contexto ambiental y económico) en cuanto a la disponibilidad y calidad de los recursos, dentro de un equilibrio que supera lo meramente ecológico (pero que lo incluye). Esto en relación a las expectativas comunitarias, pero sin olvidar que dichas expectativas vienen conformadas por un marco ideológico referente o dominante.

Gómez López (1994) sostiene que la calidad de vida se determina a partir del nivel de vida, compuesto por el ingreso directo (dinero que reciben los individuos por su trabajo ya sea salario o jubilación) e indirecto (dinero que reciben los miembros de una sociedad organizada como la educación, salud, seguridad, justicia, entre otros), sumado a la calidad ambiental. Esta última involucra un orden existencial o funcional -de carácter cuantitativo- que incluye el equipamiento urbano, infraestructura, accesibilidad, estado de las edificaciones, entre otros, y un orden simbólico y representativo - de carácter cualitativo- que incluye elementos de significación que están en la memoria colectiva y que satisfacen necesidades de identidad y legibilidad urbana.

A su vez, la calidad de vida es definida por Velázquez (2001) como una medida de logro en función de un techo, umbral máximo o un nivel establecido como óptimo, teniendo en cuenta dimensiones socioeconómicas y ambientales que dependen de una escala de valores sociales y que varían en función de las expectativas sociales e individuales del progreso histórico.

Casas (1996) la define como una integración entre los indicadores sociales y del medio ambiente considerando el bienestar, no sólo como dependiente de las cuestiones materiales (indicadores económicos) sino también de los valores humanos (indicadores sociales). Este autor se refiere a las percepciones, aspiraciones, necesidades, satisfacciones y representaciones sociales que los miembros de todo conjunto social experimentan en relación a su entorno y dinámica social en la que se encuentran inmersos, incluyendo los servicios e intervenciones sociales de las que son destinatarios y que provienen de las políticas sociales.

Para Tonon y Castro Solano (2012) la calidad de vida se asocia al bienestar social referido al entorno material y al bienestar psicológico referido al entorno psicosocial (experiencia y evaluación personal de la vida). De esta forma, la conceptualización de la calidad de vida posee una implicancia política en base al respeto de derechos humanos y una realidad social (Tonon, 2003; en Tonon y Castro Solano, 2012:167). Estos autores hacen referencia a cinco dimensiones de la calidad de vida a nivel nacional, poniendo énfasis en (1) las acciones del gobierno como nivel de respuesta de las decisiones gubernamentales a las necesidades de la población, la transparencia en las decisiones gubernamentales, la situación económica general, la seguridad financiera, los planes asistenciales gubernamentales, los planes asistenciales gubernamentales en emergencias, las condiciones de seguridad pública en la vida cotidiana y las libertades políticas; (2) la salud y Educación como el sistema estatal de salud y posibilidades de acceso al sistema estatal de educación; (3) el hábitat como nivel de cuidado del medio ambiente y respeto a los espacios públicos; (4) el acceso a recursos como a la vivienda, sistema de recaudación de impuestos y empleo; y (5) las actitudes ciudadanas 
de respeto a las diferencias culturales y religiosas.

Las redes de transporte público constituyen, por lo tanto, un indicador de calidad de vida en tanto brindan un servicio esencial a la comunidad y de cohesión social urbana al contribuir en la movilidad de la población a los distintos sectores de la ciudad (vinculan la periferia con el área central y con otros centros entre sí). Sin embargo, constituyen un indicador de calidad ambiental en tanto mientras mayor es su servicio, mayor niveles de $\mathrm{CO}_{2}$ producen (Castañeda, inédito).

De este modo, analizar la movilidad urbana desde la existencia de paradigmas epocales implica comprender que si bien -temporalmente- nos encontramos en una estructura de pensamiento definida como posmodernidad, los patrones latinoamericanos de movilidad sin duda no han traspasado los modelos instaurados en la modernidad; ya que si bien el estudio sobre la movilidad urbana sostenible cada vez es mayor, las políticas públicas latinoamericanas actuales no logran un cambio de matriz energética, lo que conlleva a un costo ambiental muy alto y a ciudades poco sustentables.

Por ello, el objetivo de este trabajo consiste en identificar en el Área Metropolitana de Tucumán (en adelante $\mathrm{AMeT}$ ) como se muestra en el siguiente plano, entendida como ciudad intermedia de mayor crecimiento del Noroeste Argentino, la emisión de $\mathrm{CO}_{2}$ de la red del transporte público en relación a la cobertura de áreas verdes o presencia de actividad fotosintética que permita contrarrestar niveles altos de $\mathrm{CO}_{2}$ emitidos por el uso diario de las infraestructuras en la ciudad.

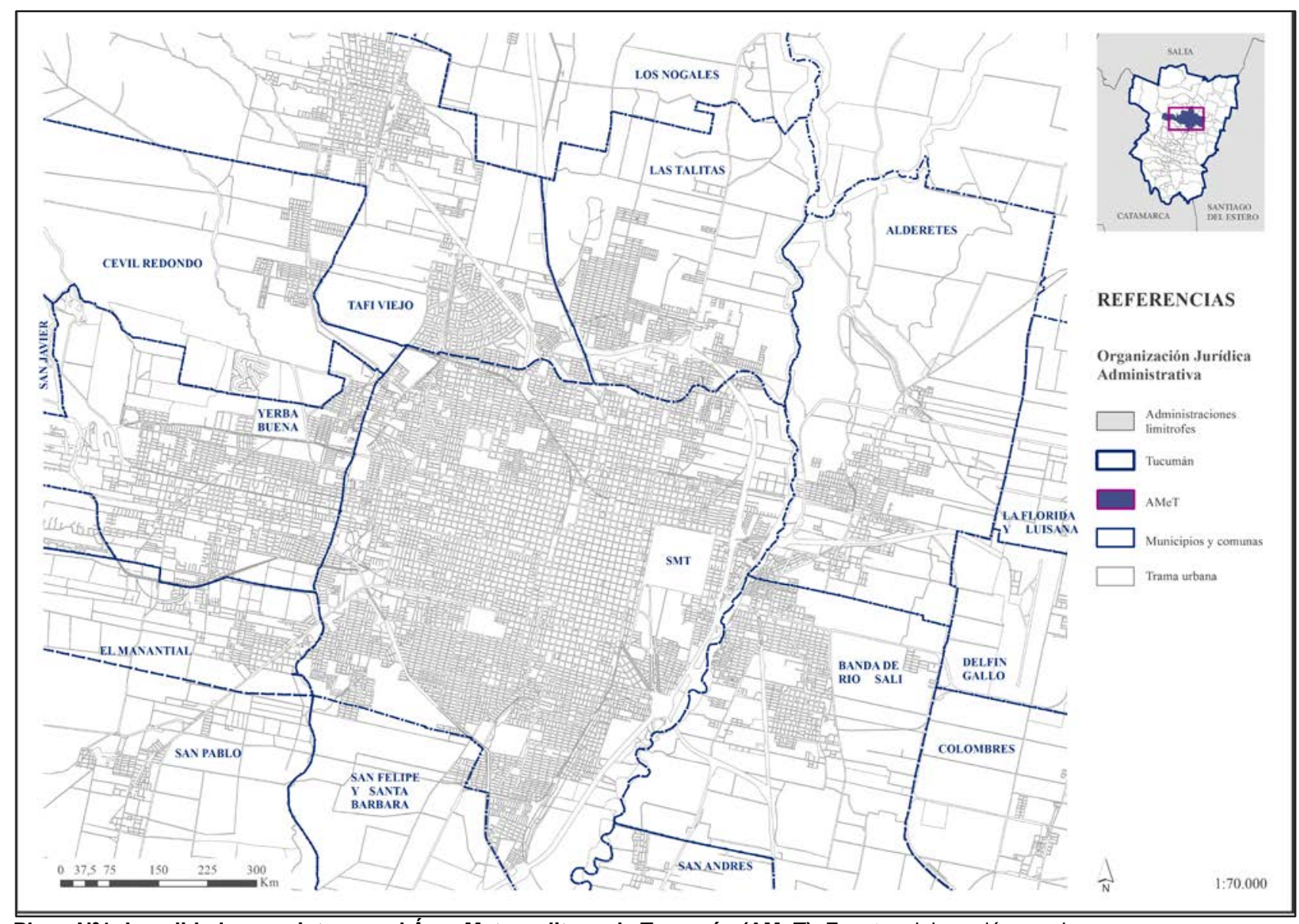

Plano №1: Localidades que integran el Área Metropolitana de Tucumán (AMeT). Fuente: elaboración propia.

\section{METODOLOGÍA}

El trabajo se lleva a cabo con una metodología mediante la utilización de Sistemas de Información Geográfica (SIG) sobre la cual se desarrollan los análisis para el período 2013-2017 y se vinculan las variables mencionadas para la obtención de áreas críticas en el AMeT que permitan la generación de propuestas sustentables de intervención. Las fases de trabajo son las siguientes:

1. Se definen las variables de análisis, las cuales fueron extraídas de la tesis doctoral de la autora (inédita): 
a. Niveles de actividad fotosintética: dichos niveles se obtienen a partir de la variable de NDVI (Índice de Vegetación de Diferencia Normalizada), la cual constituye un índice de vegetación normalizado que se relaciona con las medidas de biomasa y cuya interacción entre la vegetación y energía electromagnética, en las longitudes de ondas del rojo al infrarrojo cercano, indican niveles fotosintéticos de la vegetación que se traducen en la presencia/ausencia de clorofila y estructura de las células de las hojas (Eastman, 2012). Este indicador se considera de gran importancia en la identificación de áreas vulnerables al constituir una variable que indica actividad constante fotosintética de la vegetación y, por lo tanto, absorción de $\mathrm{CO}_{2}$; de manera que, a partir de la vinculación de las bandas rojo e infrarrojo cercano, se obtienen gradientes en la coloración del suelo que evidencian la cobertura de suelo más árido (cuando es baja) y de mayor nivel de vegetación (cuando es alta). Como criterio de selección se tiene en cuenta una Imagen Satelital Sentinel-2, de captura del 17 de diciembre del año 2016, en tanto constituye térmicamente- uno de los meses más desfavorables del año.

De modo que dicha variable se obtuvo de un cálculo realizado en calculadora ráster [(banda infrarrojo cercano - banda roja) / (banda infrarrojo cercano + banda roja)], del cual se extrajo, según la coloración del píxel, la localización y distribución de áreas con mayor actividad fotosintética.

b. Espacios verdes públicos: estos servicios son de carácter público y promueven la actividad deportiva, recreacional, cultural, científica, artística, turística, de esparcimiento, ocio y estancia. Dichas dotaciones son de carácter nodal al atraer población y actividades, y constituyen un indicador de calidad ambiental en tanto pueden funcionar como pulmones en las ciudades a través de absorber dióxido de carbono y emitir oxígeno. Como criterio de selección se tienen en cuenta las plazoletas, plazas, parques urbanos y metropolitanos de uso público. El relevamiento de estos servicios se llevó a cabo a partir de visores de mapas oficiales ${ }^{4}$, los cuales no permitieron su uso directo, pero sí corroborar su localización y dibujarla en una base SIG para su posterior manejo.

c. Niveles de emisión de $\mathrm{CO}_{2}$ del transporte público de pasajeros: éste servicio se integra por redes que constituyen el medio de movilidad de acceso público de la población hacia y desde las distintas zonas del área metropolitana, y a la vez, un tipo de transporte terrestre de coordinación y gestión pública municipal o provincial cuya modalidad de funcionamiento consiste en recorridos, frecuencias y tarifas estipuladas por operadores del servicio. Como criterio de selección se tienen en cuenta las líneas de recorridos de transporte público de corta y media distancia (urbanas e interurbanas) y no se tienen en cuenta -en cambio- las que exceden la jurisdicción metropolitana. Los datos de localización de la red de transporte público se obtuvo de la municipalidad de San Miguel de Tucumán (Secretaría de Planeamiento Urbano). Luego de su relevamiento en formato Dwg. se traspasaron los recorridos a una base SIG, con excepción de las líneas 109 y 142 cuyo recorrido se obtuvo de un servidor web alternativo ${ }^{5}$ debido a su inexistencia en la base de datos anterior.

La variable de emisión de $\mathrm{CO}_{2}$ del transporte público de pasajeros se integra por la cobertura de red de dicha infraestructura y constituye un indicador de presencia constante que expresa la emisión de dióxido de carbono del consumo del transporte público urbano. Se considera una variable de gran importancia en la calidad ambiental en tanto constituye una fuente de energía que circula con gran intensidad sobre la trama de la mayoría de las ciudades del mundo, y en el caso de Tucumán, constituye el único medio de movilidad pública para la población.

Como criterio de selección, se tuvo en cuenta la densidad de emisión de $\mathrm{CO}_{2}$ en función de la cobertura de dicha red y su respectivo funcionamiento medido en frecuencias de uso. Su cálculo se llevó a cabo teniendo en cuenta la metodología de la Secretaría de Ambiente y Desarrollo Sustentable de la Nación Argentina ${ }^{6}$. De esta manera se procedió a la vinculación de los datos de emisión (mediante su incorporación a las tablas alfanuméricas de los SIG) con las redes de transporte relevadas (disponibles en formato vector) para luego convertir dichos datos a un mapa de densidad y posteriormente, representarlo en gradientes de emisión.

2. En función de la identificación de áreas con NDVI, se clasifican los espacios verdes públicos relevados según posean o no actividad fotosintética. De este paso se obtienen las dotaciones públicas potenciales a absorber el $\mathrm{CO}_{2}$ proveniente del uso de las infraestructuras de transporte.

\footnotetext{
${ }^{4}$ Visores de mapas de espacios verdes: (http://idet.tucuman.gob.ar/), (http://central.tucuman.gov.ar:8180/pmapper-dev/map.phtml) y (http://smt.gob.ar/lugares).

${ }_{5}$ Servidor web alternativo: (http://tucubondi.com.ar/recorridos)

${ }^{6}$ Cálculo de emisión de $\mathrm{CO}_{2}$ en función de la cobertura de transporte público: [número de viajes (por día) x recorrido de viaje (en km.) x 1 / eficiencia del combustible $(16.66 \mathrm{Km} /$ litros $) \times$ el factor de emisión del gasoil $\left(2.77 \mathrm{KgCo}_{2} /\right.$ litros $\left.)\right]$ / [1000 x carga promedio del colectivo (20 pasajeros) $]=\mathrm{Tn}$. de $\mathrm{CO}_{2}$ diarios por habitante.
} 
3. Se cruza la variable de espacios verdes públicos (con y sin actividad fotosintética) con la de emisión de $\mathrm{CO}_{2}$ del transporte público y se observan las distintas situaciones (niveles de calidad ambiental) existentes en el AMeT. En este paso se identifican aquellos espacios verdes públicos que se encuentran en condiciones críticas, situación que posibilita generar un criterio de intervención (priorización), desde la política pública, para la revitalización de dichas dotaciones y disminución de la huella de carbono urbana.

\section{RESULTADOS}

\section{1 a Niveles de actividad fotosintética en el AMeT}

El aglomerado de Tucumán posee, casi en su totalidad, los niveles más bajos de NDVI, situación que puede asociarse no solo a la existencia de suelo con escasa dotación verde o aquella que no absorbe $\mathrm{CO}_{2}$, sino también a suelo agrícola cuyo cultivo tampoco genera absorción.

Existe una diferencia, como se muestra en el plano №2, entre la capital provincial de San Miguel de Tucumán (SMT), las localidades situadas al este (Banda de Río Salí, Alderetes, La Florida y Luisana, Delfín Gallo, Colombres, San Andrés y San Felipe y Santa Bárbara), oeste en contacto con la sierra de San Javier (Yerba Buena, Tafí Viejo, El Manantial, San Pablo, San Javier y Cevil Redondo) y noroeste del aglomerado (Las Talitas y Los Nogales), en tanto éstas últimas poseen mayor proporción de actividad fotosintética.

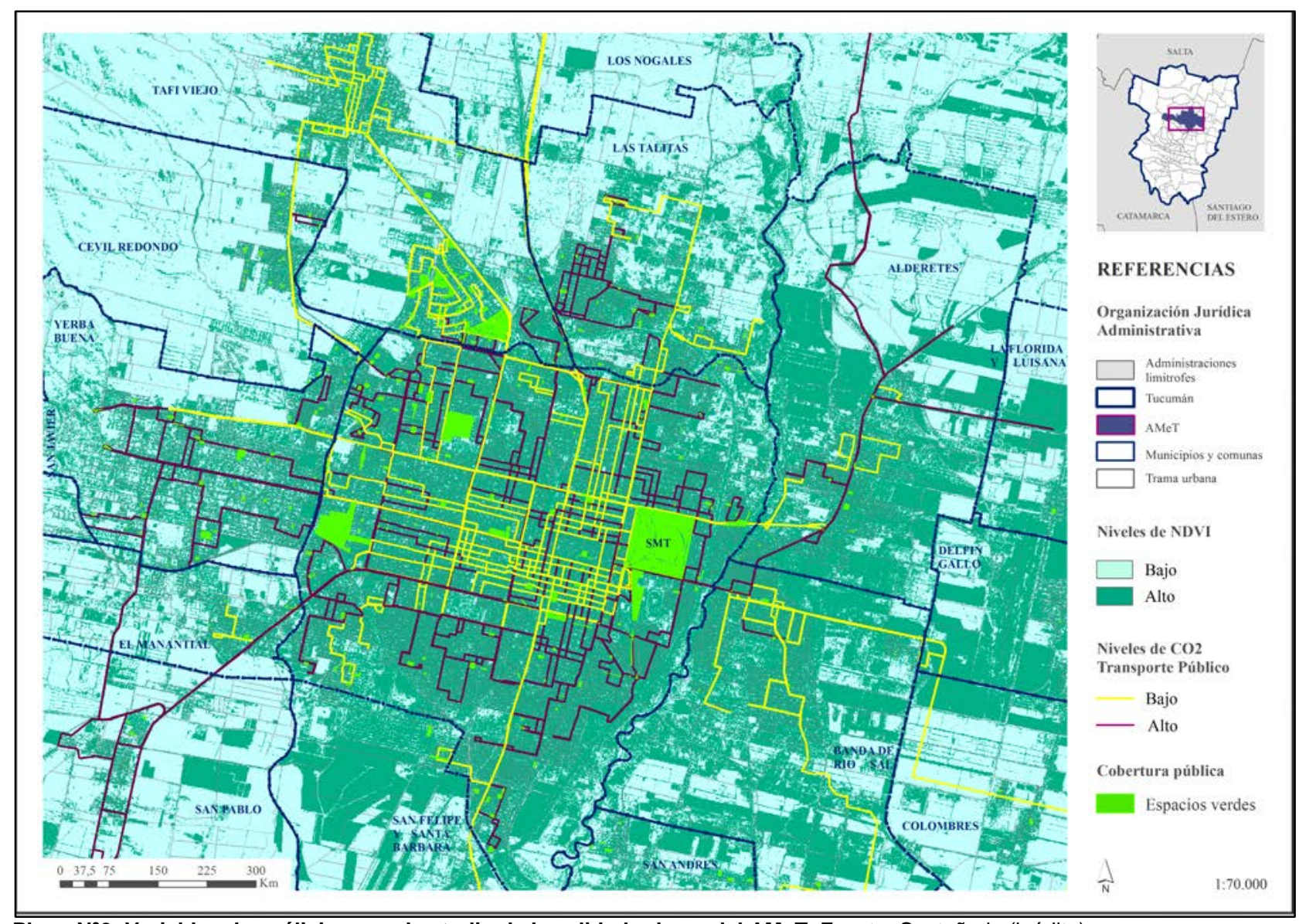

Plano N²: Variables de análisis para el estudio de la calidad urbana del AMeT. Fuente: Castañeda (inédito).

En el caso de San Miguel de Tucumán, salvo sectores con localización de parques urbanos y otras áreas al noreste y este, posee en casi toda su superficie baja actividad fotosintética.

El municipio de Yerba Buena, en cambio, posee sectores con nivel bajo intercalados con otros de nivel alto, y éstos últimos se encuentran fundamentalmente ubicados al norte, oeste y sureste de dicha localidad.

Las comunas de El Manantial, San Pablo, Cevil Redondo, Los Nogales y Colombres, si bien concentran áreas con nivel bajo de NDVI en concordancia con la expansión urbana y algunos cultivos, poseen mayor proporción de áreas con altos niveles.

El municipio de Tafí Viejo posee incidencia intermedia de ambos, coincidiendo los niveles bajos con el área urbanizada y los altos con áreas verdes localizadas al este y oeste de dicha administración. Lo mismo sucede 
en Las Talitas donde áreas con menores niveles se ubican en sectores de expansión urbana, mientras que los mayores niveles al norte, este y oeste de la misma.

El municipio de Banda de Río Salí posee casi la totalidad de su superficie con niveles bajos de NDVI; al igual que Alderetes y La Florida y Luisana, aunque éstos poseen niveles altos al norte de su jurisdicción.

Delfín Gallo, San Andrés y San Felipe y Santa Bárbara, si bien constituyen comunas con suelo mayormente destinado a cultivo y escasa área urbanizada, tienen -en mayor proporción- niveles bajos de NDVI.

\section{1 b Dotación metropolitana de espacios verdes públicos}

Se relevaron, teniendo en cuenta un recuento de unidades, un total de 451 equipamientos verdes públicos, de los cuales, el $98 \%$ se constituye de plazas y plazoletas, el $1.5 \%$ de parques urbanos y el $0.5 \%$ de parques metropolitanos.

De las localidades que integran el AMeT, San Miguel de Tucumán concentra más del $50 \%$ de cobertura del total de equipamientos, como se muestra en el plano $\mathrm{N}^{0} 2$, mientras que el resto de administraciones no superan el 15\%; siendo los casos más desfavorables los de las comunas de San Pablo, Cevil Redondo, La Florida y Luisana, Delfín Gallo, Los Nogales, San Felipe y Santa Bárbara y San Javier (al no superar el 1\%). Lo mismo sucede si se desglosa del total la incidencia de plazas y plazoletas por localidad. Aunque en el caso de los parques casi el $80 \%$ se localiza en la capital y el porcentaje restante distribuido de igual manera entre Yerba Buena y Tafí Viejo. Entre éstas localidades que poseen cobertura de parques, es posible diferenciar los urbanos (que suman un total de 7 unidades), de los metropolitanos (que suman un total de 2 unidades) desde el nivel de alcance y extensión en superficie, excediendo en el segundo caso las 50 hectáreas de dimensión?.

En el caso de San Miguel de Tucumán, Yerba Buena y Tafí Viejo, poseen mayor cantidad de plazas y plazoletas, y menor de parques (con mayor incidencia de la capital sobre el resto).

Alderetes, Las Talitas, Cevil Redondo, San Andrés, San Pablo y El Manantial poseen mayor cantidad de plazas y plazoletas; mientras que Banda de Río Salí y La Florida y Luisana poseen menor proporción. Sin embargo todas ellas poseen nula cobertura de parques.

\section{1 c Emisión de $\mathrm{CO}_{2}$ de la red de transporte público de pasajeros}

La infraestructura de transporte público de pasajeros cubre casi toda la superficie de suelo del AMeT, aunque se concentra en mayor medida en áreas centrales y se encuentra dispersa en áreas periféricas de cada localidad.

Se relevaron 32 circuitos urbanos que solo operan dentro de San Miguel de Tucumán y 48 interurbanos que operan desde éste hacia el resto del aglomerado. Esto evidencia que la capital concentra el total de líneas urbanas y 45 de las interurbanas, con excepción de un recorrido de la línea 100 que no incluye al municipio capital. En el resto de administraciones que solo poseen recorridos interurbanos -y teniendo en cuenta que un circuito puede contener varias administraciones- la cobertura de transporte público es menor, como es el caso de Yerba Buena con una cantidad de 13 líneas; Banda de Río Salí con 11; Tafí Viejo con 10; Alderetes y El Manantial con 8; San pablo con 5; Las Talitas con 4; Cevil Redondo con 3; San Felipe y Santa Bárbara y Delfín Gallo con 2; Los Nogales, La Florida, y Cebil Redondo con 1; y nula en San Javier y San Andrés.

En relación a las áreas con mayor emisión de $\mathrm{CO}_{2}$, éstas coinciden con vías de rápida circulación (fundamentalmente rutas y avenidas) del área metropolitana y específicamente con áreas en las que confluyen recorridos urbanos e interurbanos, como se muestra en el plano $\mathrm{N}^{0} 2$.

Teniendo en cuenta que la emisión de $\mathrm{CO}_{2}$ se encuentra directamente relacionada a los kilómetros recorridos y frecuencia de las redes de transporte público, si se analiza el porcentaje de $\mathrm{CO}_{2}$ emitido por día por tipo de localidad del AMeT, se observa que San Miguel de Tucumán concentra más del $80 \%$ de emisión, mientras que el resto no supera el 5\%; siendo los casos más favorables los de las comunas de Cevil Redondo, Colombres, San Felipe y Santa Bárbara, Los Nogales, La Florida y Luisana, Delfín Gallo (que no superan el $1 \%)$, San Javier y San Andrés (sin emisión).

\section{Actividad fotosintética de los espacios verdes públicos del AMeT}

Del total de equipamientos verdes públicos relevados, teniendo en cuenta un recuento de unidades, se

\footnotetext{
${ }^{7}$ Los parques metropolitanos reconocidos que se localizan en SMT se constituyen por el 9 de Julio y Guillermina. Mientras que los parques urbanos distribuidos en SMT se constituyen por el Avellaneda, Centenario, Campo Norte, Mercedes Sosa y del Rey; en YB por el Percy Hill; y en TV por el Parque Sur, con mayor proporción por parte de SMT.
} 
identifica un $68 \%$ sin actividad fotosintética, como se muestra en el plano №3. De éstos, el caso más desfavorable se constituye por San Miguel de Tucumán, el cual concentra un $60 \%$ del total; mientras que el resto de localidades no supera el $10 \%$. Los porcentajes más bajos se encuentran en las comunas de Los Nogales, La Florida y Luisana, San Felipe y Santa Bárbara, San Pablo, Delfín Gallo y Colombres. Si se tiene en cuenta dicha cobertura sin actividad fotosintética, en función del total de dotaciones que posee cada localidad, los casos más desfavorables se encuentran en Banda de Río Salí, Alderetes, San Miguel de Tucumán, La Florida y Luisana, Delfín Gallo y El Manantial; en tanto poseen mayor proporción de dotaciones sin actividad que con presencia de ella.

Del 32\% de equipamientos verdes públicos que sí posee actividad fotosintética, los casos más favorables se constituyen por San Miguel de Tucumán, Tafí Viejo y Yerba Buena, los cuales concentran más del $70 \%$ del total de éstos. Mientras que del 30\% restante los casos más desfavorables se integran por Banda de Río Salí, La Florida y Luisana, Delfín Gallo, Alderetes, Colombres, San Felipe y Santa Bárbara y El Manantial. Si se tiene en cuenta dicha cobertura con actividad fotosintética, en función del total de dotaciones que posee cada localidad, los casos más favorables se encuentran en Tafí Viejo, Los Nogales, Cevil Redondo y San Pablo; en tanto poseen mayor proporción de dotaciones con actividad que sin presencia de ella.

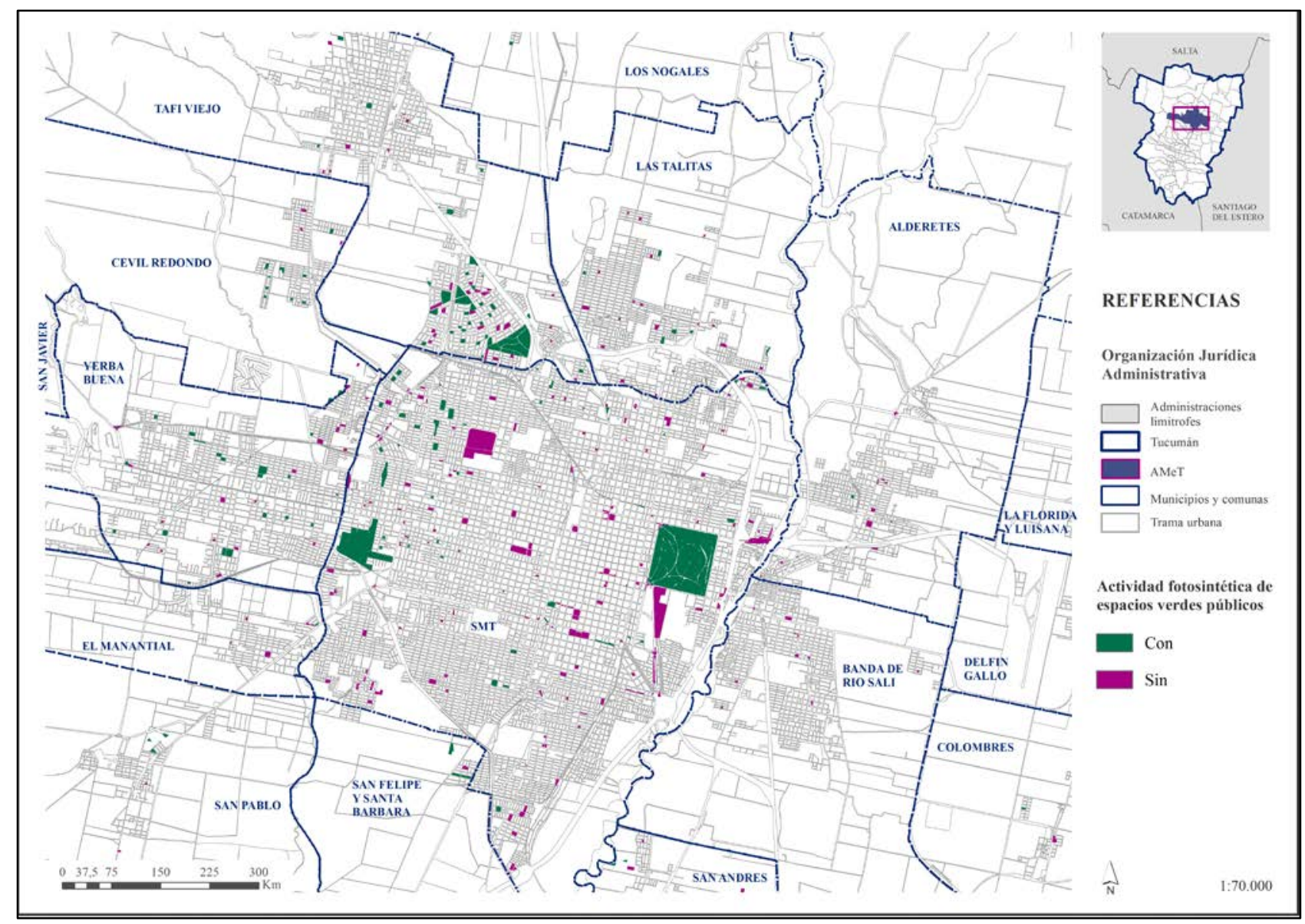

Plano №3: Espacios verdes públicos del AMeT con y sin actividad fotosintética. Fuente: elaboración propia.

\section{Situaciones de criticidad en base a dotaciones verdes públicas y capacidad de absorción de $\mathrm{CO}_{2}$.}

En función de la vinculación de las variables de equipamientos verdes públicos (según capacidad de ABSORCIÓN) y los niveles de EMISIÓN de $\mathrm{CO}_{2}$ del transporte público de pasajeros (según proximidad a éstos), es posible diferenciar seis situaciones, como se muestra en el plano №4:

Dotación verde $\mathrm{CON}$ absorción de $\mathrm{CO}_{2}$ y niveles de emisión del transporte:
1. BAJO
2. MEDIO
3. ALTO

Dotación verde $\mathrm{SIN}$ absorción de $\mathrm{CO}_{2}$ y niveles de emisión del transporte:

4. BAJO 


\section{MEDIO \\ 6. ALTO}

Del total de equipamientos verdes públicos con capacidad de absorción (143 dotaciones), representados con las situaciones 1, 2 y 3 mencionadas previamente; más del 70\% se encuentra con bajos niveles de emisión del transporte, mientras que el $25 \%$ con nivel medio y el $1 \%$ con nivel alto.

Por otro lado, del total de equipamientos verdes públicos sin capacidad de absorción (308 dotaciones), representados con las situaciones 4,5 y 6 mencionadas previamente; más del $50 \%$ se encuentra con bajos niveles de emisión del transporte, mientras que el $43 \%$ con nivel medio y el $5 \%$ con nivel alto.

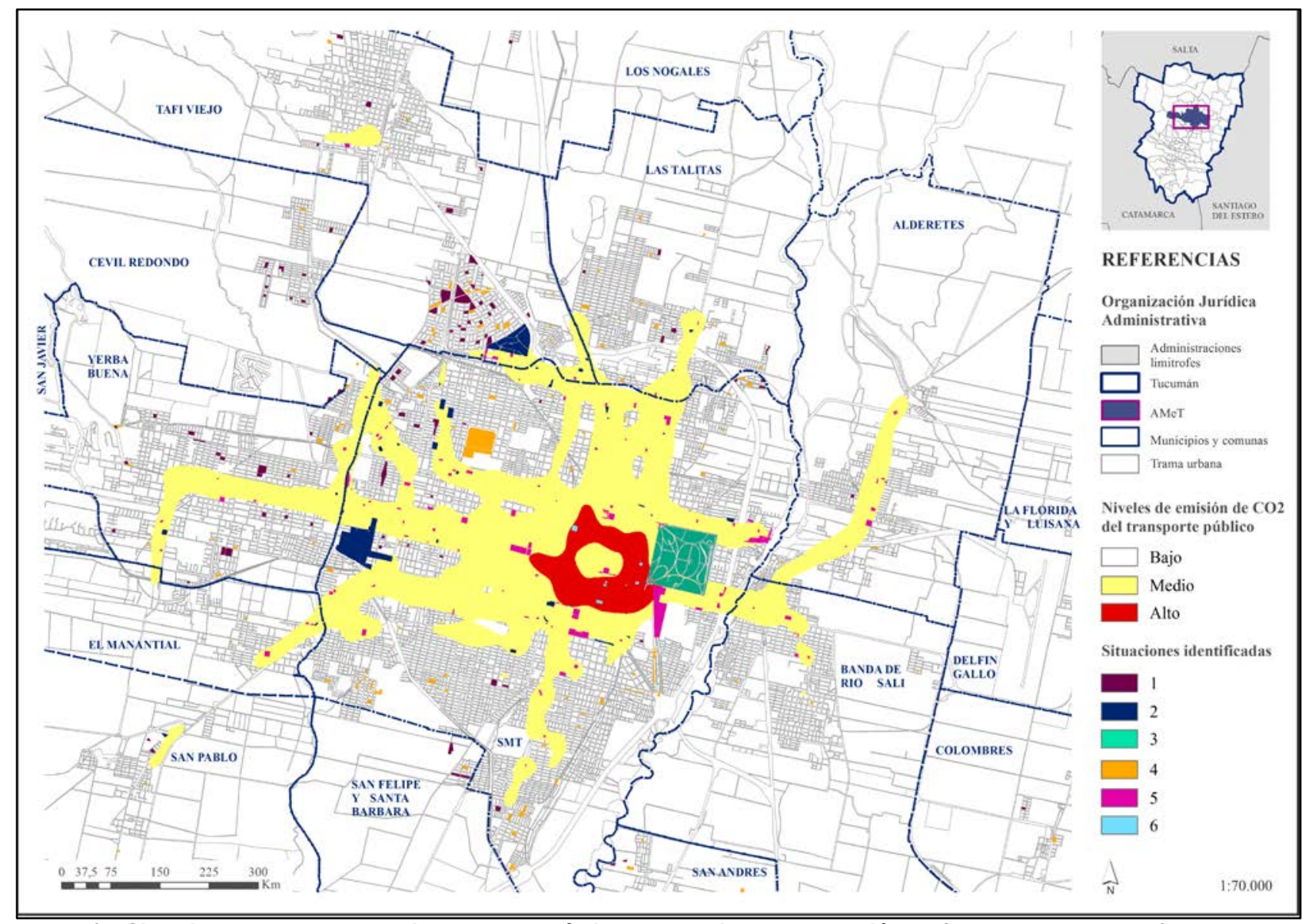

Plano ํ4: Situaciones en base a dotaciones verdes públicas y capacidad de absorción de $\mathrm{CO}_{2}$. Fuente: elaboración propia.

De las localidades del aglomerado, en San Miguel de Tucumán se observan las seis situaciones, con mayor proporción de áreas verdes sin absorción y niveles bajos y medios de emisión (4 y 5); incidencia intermedia de áreas verdes con absorción y niveles bajos y medios de emisión (1 y 2); y baja proporción de áreas verdes con y sin absorción, y nivel alto de emisión (3 y 6).

Los municipios de Yerba Buena, Tafí Viejo y Las Talitas se comportan de modo similar entre si, en tanto no poseen áreas verdes con y sin absorción en combinación con un nivel alto de emisión (3 y 6); y presentan baja incidencia del nivel medio de emisión (2 y 5). En el caso de Yerba Buena y Tafí Viejo poseen mayor proporción de áreas verdes con absorción y nivel bajo de emisión (1), e intermedia de áreas verdes sin absorción y nivel medio de emisión (4); mientras que Las Talitas posee mayor proporción de áreas verdes sin absorción y nivel medio de emisión (4) e intermedia de áreas verdes con absorción y nivel bajo de emisión (1).

Las localidades de Alderetes y El Manantial tampoco poseen áreas verdes con y sin absorción en combinación con un nivel alto de emisión (3 y 6), aunque presentan mayor proporción con nivel medio de emisión e intermedio con bajo. Sin embargo, Alderetes posee baja incidencia de áreas verdes con absorción y nivel bajo (1), y nula con nivel medio (2); mientras que El Manantial posee baja incidencia de áreas verdes con capacidad de absorción y nivel medio (2), y nula con bajo (1).

En las comunas de San Pablo y San Felipe y Santa Bárbara no se observan áreas verdes con absorción y nivel alto de emisión (3), como tampoco áreas verdes sin absorción y nivel medio y alto de emisión (5 y 6 ). Sí poseen, en cambio, mayor proporción de áreas verdes con y sin capacidad de absorción y nivel bajo (1 y 4); 
aunque San Pablo posee incidencia intermedia de áreas con absorción y nivel medio (2), mientras que San Felipe y Santa Bárbara posee incidencia nula en dicha situación.

Por su parte Banda de Río Salí no posee áreas con absorción (1, 2 y 3), ni tampoco dotaciones sin absorción y nivel alto de emisión (6); aunque posee, en el caso de espacios verdes sin absorción, mayor proporción con nivel medio e intermedio con bajo.

Las comunas de Colombres y San Andrés también se comportan de modo similar entre sí, ya que ambas poseen ausencia de áreas verdes con ambos tipos de absorción y niveles medios y altos de emisión (2, 3, 5 y 6); como así también, mayor proporción de dotaciones sin absorción y nivel bajo (4) e intermedia de dotaciones con absorción y nivel bajo (1).

Las localidades de Los Nogales, La Florida y Luisana, y Delfín Gallo tampoco cuentan, como en el caso anterior, con las situaciones 2, 3, 5 y 6; con la diferencia de que Los Nogales posee áreas con absorción y nivel bajo de emisión (1) y nula cantidad de áreas sin absorción y nivel bajo de emisión (4), mientras que La Florida y Luisana y Delfín Gallo poseen la situación contraria (nivel bajo en situación 4 y nula en 1).

En relación a lo expuesto anteriormente, se clasifican las seis situaciones identificadas en 4 categorías de criticidad de calidad ambiental, como se muestra en el plano №5:

1. Criticidad ambiental alta: situación 6

2. Criticidad ambiental intermedia: situaciones 3 y 5

3. Criticidad ambiental baja: situación 2

4. Sin criticidad ambiental: situaciones 1 y 4

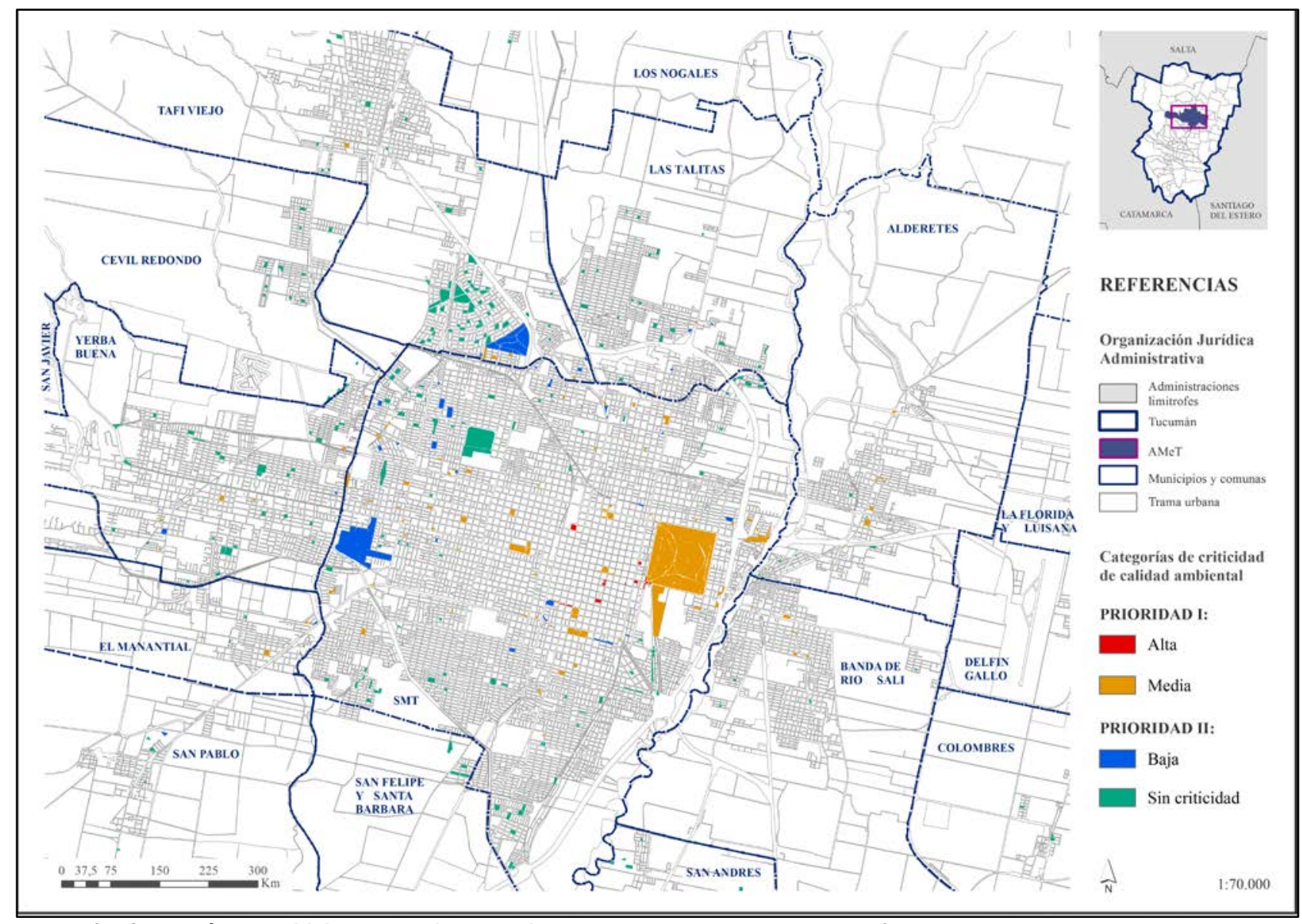

Plano ํ5: Categorías de criticidad de calidad ambiental en el AMeT. Fuente: elaboración propia.

\section{CONCLUSIONES}

Los resultados alcanzados permiten evidenciar la importancia del transporte público como indicador de medición de huella de carbono y servicio de acceso de la población al uso de la ciudad y de los espacios verdes como servicios ambientales que permiten una mayor adaptación al cambio climático; pero a la vez, muestra la importancia de incorporar medidas complementarias desde las políticas públicas y desde el cambio de los modelos territoriales actuales insustentables para aumentar las condiciones de habitabilidad y calidad de vida de la población en las ciudades. 
Entre los resultados más significativos se encuentra que los espacios verdes públicos con capacidad de absorción de $\mathrm{CO}_{2}$ son escasos. En la mayoría de los casos las áreas de influencia de dichos espacios poseen mayor absorción, lo cual incentiva a pensar que las áreas verdes privadas poseen mayor actividad fotosintética que las públicas. Por otro lado, no se observa una correlación entre la superficie de área verde pública y los niveles de absorción, en tanto en muchos casos las de menor dimensión poseen mayor superficie de ésta.

En el caso del transporte público, las áreas con menor emisión enmascaran la falta de cobertura de dicho servicio fundamentalmente en la periferia y sectores de expansión urbana de las últimas décadas, situación que se traduce en una movilidad que actúa como ineficiente frente a los requerimientos actuales de traslado de la población hacia los centros urbanos. Tal es el caso de las dotaciones con situación 1, que se traducen en sectores con baja cobertura de transporte público donde el $\mathrm{CO}_{2}$ emitido está directamente asociado al recorrido y cantidad de líneas existentes.

La dotación del espacio verde público no mantiene actualmente relación directa con las áreas donde se localiza la mayor emisión de $\mathrm{CO}_{2}$ por transporte público de pasajeros. En este sentido, no se observan plazas y parques con actividad fotosintética y niveles altos de emisión del transporte público, salvo en el caso del Parque 9 de Julio, el cual se encuentra rodeado por los niveles más altos de emisión de toda el área metropolitana.

Se identifican como los casos más desfavorables de calidad ambiental, las dotaciones verdes públicas localizadas en San Miguel de Tucumán, Banda de Río Salí, Alderetes y El Manantial; y las menos desfavorables las situadas en Yerba Buena, Tafí Viejo, Las Talitas, Los Nogales, La Florida y Luisana, Delfín Gallo, Colombres, San Andrés, San Felipe y Santa Bárbara, Cevil Redondo y San Pablo. En éste último caso, algunas de las comunas poseen baja criticidad de calidad ambiental por su bajo nivel de urbanidad, esto es su escaso desarrollo en materia de acceso a servicios administrativos, culturales, sanitarios y educativos de nivel superior.

Por otro lado, en función de los resultados se elaboran dos categorías según su prioridad de intervención, como se muestra en el plano N05: una TIPO I que engloba las dotaciones verdes públicas con categorías de criticidad 1 y 2, en tanto enmascaran su nula planificación estatal en cuanto a su localización en áreas con mayores requerimientos de absorción de $\mathrm{CO}_{2}$ y una mayor vulnerabilidad de la población residente por los niveles bajos de calidad ambiental de dichas áreas de influencia; y una categoría TIPO II que engloba las categorías 3 y 4 , ya que al poseer baja emisión del transporte no constituyen un problema para la población residente, y en los casos en los que las dotaciones poseen actividad fotosintética, éstos contribuyen en la oxigenación urbana. Se entiende, por lo tanto, que la primera categoría podría adquirir una mayor urgencia de gestión en términos de tratamiento o mejora de las condiciones de vida de la población residente o desde el planteo de prioridades de intervención estatal.

Se observa, de esta forma, que algunas localidades más desfavorables identificadas en el AMeT coinciden con las áreas de mayor centralidad y accesibilidad urbana ya que en éstas los requerimientos de movilidad son mayores. En este sentido, que dichas áreas se encuentren con niveles bajos de calidad ambiental constituye una gran contradicción, ya que si no mejoran sus condiciones quedan postergadas o ligadas a un uso insustentable de las intervenciones urbanas, donde las áreas verdes están lejos de funcionar como pulmones urbanos.

En este contexto, se observa un desfasaje entre la dotación estatal de equipamientos verdes públicos y la generación de bienestar social urbano, ya que en vez de utilizar un criterio de dotación de servicios, localizan a éstos de manera aleatoria; situación que es necesaria replantear si se quiere un modelo de ciudad con mayores niveles de calidad urbana.

\section{BIBLIOGRAFÍA}

ARDILA, R. (2003). Calidad de vida: una definición integradora. Revista Latinoamericana de Psicología, vol. $35, N^{\circ} 2,161-164$.

BLANCO, J., BOSOER, L., APAOLAZA, R. (2014). Gentrificación, movilidad y transporte: aproximaciones conceptuales y ejes de indagación. Revista de Geografía Norte Grande, vol. 58, 41-53. 
BOLDRINI, P.; DEL CASTILLO, A.; MALIZIA, M. Condiciones de vida y fragmentación socio-espacial en el Aglomerado Gran San Miguel de Tucumán (noroeste argentino). Revista Estudios Socioterritoriales, 2014, vol. 15, 15-43. Disponible en: <http://www.scielo.org.ar/scielo.php?script=sci_arttext\&pid=S1853$43922014000100002 \&$ lng=es\&nrm=iso $>$.

CASAS, F. (1996). Bienestar Social. Una Introducción Psicosociológica. Barcelona: PPU.

CASTAÑEDA, A. L. (2017). La infraestructura de gas como indicador de habitabilidad urbana: el caso del Área Metropolitana de Tucumán. Revista Urbana, No34, 16-25.

CASTAÑEDA, A. L. (inédito). Metodología de detección de umbrales ambientales en base a los servicios urbanos. Un aporte a la evaluación del área metropolitana de Tucumán. Tesis Doctoral en Ciencias Sociales. Universidad Nacional de Tucumán, Argentina.

CELEMín, J. P. (2007). El estudio de la calidad de vida ambiental: definiciones conceptuales, elaboración de índices y su aplicación en la ciudad de Mar del Plata, Argentina. Revista Hologramática, 06(07), 71-98.

CHOMBAR DE LAUWE, M. J. (1976). L'apropiation de l'espace par les enfants i precessus de socialisation. In $\mathrm{P}$. Corosec. Apropiation de l'espace. Actes de la tressième conference international de psycologie de l'espace construite. Strasbourg, France.

DANGOND GIBSONE, C., FRANCOIS JOLLY, J., MONTEOLIVA VILCHES, A., ROJAS PARRA, F. (2011). Algunas reflexiones sobre la movilidad urbana en Colombia desde la perspectiva del desarrollo humano. Revista Pap. Polít. Bogotá, vol. 16, №2, 485-514.

DI LULLO, R. (2009). El espacio público urbano: temas de estudio.Tucumán: Editorial EDUNT Universidad Nacional de Tucumán.

FIGUEROA, O. (2005). Transporte urbano y globalización. Políticas y efectos en América Latina. Revista Eure, vol. XXXI, num. 94, pp. 41-53. Disponible en: http://www.scielo.cl/pdf/eure/v31n94/art03.pdf

GÓMEZ LÓPEZ, R. (1994). Apuntes de Clases de la Cátedra de Urbanismo II [Material de clase]. Urbanismo II. Instituto de Planeamiento y Desarrollo Urbano, Facultad de Arquitectura y Urbanismo, Universidad Nacional de Tucumán, Argentina.

GUTIÉRREZ, A. (2000). La producción del transporte público en la metrópolis de Buenos Aires: Cambios recientes y tendencias futuras. Revista EURE, vol. 26, № 77, 109-136.

HERNÁNDEZ AJA, A. (2009). Calidad de vida y medio ambiente urbano: Indicadores locales de sostenibilidad y calidad de vida. Revista INVI, 24(65), 79-111.

HERNÁNDEZ AJA, A.; LEIVA RODRÍGUEZ, A. (2006). Parámetros dotacionales para la ciudad de los ciudadanos. Madrid: Editorial Cuadernos del Instituto Juan de Herrera.

KUHN, T. (1971). La estructura de las revoluciones científicas. México: Fondo de la Cultura Económica. RODRÍGUEZ VIGNOLI, J. (2008). Movilidad cotidiana, desigualdad social y segregación residencial en cuatro metrópolis de América Latina. Revista Eure, vol. XXXIV, núm. 3, pp.49-71. Disponible en: http://dx.doi.org/10.4067/S0250-71612008000300003.

LONGHI, F.; BOLSI, A.; VELÁZQUEZ, G.; PAOLASSO, P.; CELEMÌN, J. P. (2015). Fragmentación socioterritorial y condiciones de vida en Argentina en los albores del siglo XXI. Revista Latinoamericana de Población, № 12, 99-131. Disponible en: <http://revistarelap.org/ojs/indexphp/relap/article/view/32>.

PARDO, R. (2000). Verdad e historicidad. El conocimiento científico y sus fracturas. En E. Díaz. La Posciencia. El conocimiento científico en las postrimerías de la modernidad (pp. 37-62). Buenos Aires: Editorial Biblos.

SECRETARIA DE AMBIENTE Y DESARROLLO SUSTENTABLE (2008). Documento de referencia: la huella de carbono del argentino promedio. Buenos Aires: Dirección de Cambio Climático-SAyDS. http://www.ambiente.gov.ar/archivos/web/UCC/File/030608_metodologia_huella_carbono.pdf 
SECRETARÍA DE ENERGÍA (2010). Cálculo del factor de emisiones de CO2 de la red argentina de energía eléctrica 2010. Argentina. http://energia3.mecon.gov.ar/contenidos/verpagina.php?idpagina=2311

LANGE VALDÉS, C. (2011). Dimensiones culturales de la movilidad urbana. Revista INVI, vol. 26, núm.71, pp.87-106. Disponible en: http://revistainvi.uchile.cl/index.php/INVl/article/view/531/562

LEVI L. Y ANDERSON, L. (1980). La Tensión Psicosocial. Población, Ambiente y Calidad de Vida. México: El Manual Moderno.

NAVARRETE RODRÍGUEZ, P. E., Y ANDRADE VALLEJO, M. A. (2010). La infraestructura del transporte público urbano en la ciudad de México y su relación con las políticas públicas. Revista Investigación Administrativa, vol. 39, №105, 41-59.

OBREGÓN BIOSCA, S. A., Y BETANZO QUEZADA, E. (2015). Análisis de la movilidad urbana de una ciudad media mexicana, caso de estudio: Santiago de Querétaro. Revista Economía, Sociedad y Territorio, vol. XV, núm. 47, pp. 61-98. Disponible en: http://www.redalyc.org/pdf/111/11132816004.pdf

PAOLASSO, P.; DEL CASTILLO, A.; MALIZIA, M.; BOLDRINI, P. (en prensa). Vulnerabilidad e informalidad urbana en el Gran San Miguel de Tucumán (NW Argentina). En Sandia Rondón, L. A. (ed.). Grandes ciudades latinoamericanas: informalidad y pobreza, viejas y nuevas formas y sus impactos en la gobernabilidad.

RODRÍGUEZ VIGNOLI, J. (2008). Movilidad cotidiana, desigualdad social y segregación residencial en cuatro metrópolis de América Latina. Revista Eure, vol. XXXIV, №3, 49-71.

SATRIANO, C. (2006). Pobreza, Políticas Públicas y Políticas Sociales. Revista Mad, №15, 60-73.

TONÓN, G. Y CASTRO SOLANO, A. (2012). Calidad de vida en Argentina: percepciones macro y micro sociales. Revista Estudios Políticos, 27, 157-171.

VELÁZQUEZ, G. (2001), Geografía, calidad de vida y fragmentación en la Argentina de los noventa. Análisis regional y departamental utilizando SIG's. Tandil, Argentina: Centro de Investigaciones Geográficas, Universidad Nacional del Centro de la Provincia de Buenos Aires.

VELÁZQUEZ, G., Y CELEMín, J. P. (2013). La Calidad Ambiental en la Argentina: análisis regional y departamental 2010. Buenos Aires, Argentina: Universidad del Centro de la provincia de Buenos Aires. 